\title{
DEEP LEARNING FOR AUTOMATIC RF-MODULATION CLASSIFICATION
}

\author{
M. Dima ${ }^{1, a}$, T. Dima ${ }^{1}$ \\ ${ }^{1}$ Univ. of Bucharest, Bucharest, Romania \\ E-mail: ${ }^{a}$ maria.dima@s.unibuc.ro
}

Classical methods use statistical-moments to determine the type of modulation in question. This essentially correct approach for discerning amplitude modulation (AM) from frequency modulation (FM) fails for more demanding cases such as AM vs. AM-LSB (lower side-band rejection) - radio signals being richer in information than statistical moments. Parameters with good discriminating power were selected in a data conditioning phase and binary deep-learning classifiers were trained for AM-LSB vs. AM-USB, FM vs. AM, AM vs. AM-LSB, etc. The parameters were formed as features, from wave reconstruction primary parameters: rolling pedestal, amplitude, frequency and phase. Very encouraging results were obtained for AM-LSB vs. AM-USB with stochastic training, showing that this particularly difficult case (inaccessible with stochastic moments) is well solvable with multi-layer perceptron (MLP) neuromorphic software.

Keywords: RF modulation, MLP neuromorphic software

Maria Dima, Mihai-Tiberiu Dima

Copyright (C) 2021 for this paper by its authors. Use permitted under Creative Commons License Attribution 4.0 International (CC BY 4.0). 


\section{Introduction}

Signals Intell (SIGINT) is a branch of the military and civilian intell services monitoring (mainly) the RF communications. Automated Modulation Classifiers (AMC's) are of 2 types: likelihood classifiers (LC's) [1] and feature classifiers (FC's) [2]. LC's use a likelihood function on the received signal, while FC's neuromorphic software for feature extraction. LC methods have high CPU demand and need prior information from transmitters. FC's do not require this, however perform relatively well. They consist of (i) feature extraction - parameters constructed from amplitude, frequency, and phase distributions [3]. Features from advanced processing, such as Fourier (FFT) and wavelet transforms [4], or high-order statistical cumulants [5] require longer signal samples and are CPU intensive, for instance noise jammed signals can be analysed with the FFT of the cyclic autocorrelation function [6] and decrypted. Secondly, (ii) classification - such as: linear, k-means [7], clustering algorithms, neural software [8] and support vector machine (SVM) with kernels [9]. Typical identification purities are 95\% [10] (S/N of $0 \mathrm{~dB}$ ) for a variety of deep-learning methods, and $90 \%$ [11] $(\mathrm{S} / \mathrm{N}$ of $-10 \mathrm{~dB})$. Existing methods assume equal signal-to-noise $(\mathrm{S} / \mathrm{N})$ in the training and witness sets.

\section{Signal conditioning and feature creation}

Before attempting to constitute any particular feature quantities, it is important to foresee how the procedure would be immune to noise and jamming. The best approach is to base the features on physially understood quantities, thus the reconstruction of the fundamental wave:

where $f, p, A$ are slowly varying

Pedestal reconstruction

$$
u(t)=p+A \sin (2 \pi f t+\phi) \text { functions of time. }
$$

- for this the simplest method is to

perform the wave average between $t_{i}$ and $t_{f}$ :

$$
\langle u\rangle=p+A_{e} \sin \left(2 \pi f \frac{t_{i}+t_{f}}{2}+\phi\right) \operatorname{sinc}\left(\pi f\left(t_{f}-t_{i}\right)\right)
$$

where $\operatorname{sinc}(x)=\sin (x) / x$ and $A_{e}=A \sin c(\pi f \Delta)$ - with $\Delta$ the duration of one sample. Since the $\sin$ term does not vanish, we try to zero the sinc term. For $t_{f} t_{i}=n \Delta$, the $\mathrm{n} \cong \mathrm{m} / \mathrm{f} \Delta$, condition must be met, with $\mathrm{m} \in \mathrm{N}$. Basically $\mathrm{m}$ is scanned until the relation gives a close-enough integer (in our case $n=11$ ) and then $\mathrm{p}=\langle\mathrm{u}\rangle$. amplitude:

Amplitude reconstruction - following the same idea, we designed a similar formula for the

$$
\left\langle\delta^{2} u\right\rangle=A_{e}^{2}\left\langle\sin ^{2}\right\rangle-A_{e}^{2}\langle\sin \rangle^{2} \simeq \frac{1}{2} A_{e}^{2}
$$

Through a coincidence the "magic number" $n$ for double the frequency is very similar to $n=11$ from pedestal determination and we can use the same loop for the averages.

Frequency reconstruction - similar to amplitude, we determined frequency with an $O_{I I}$ dipolar moment, which basically differentiates the sine wave:

$$
\begin{aligned}
\left\langle u\left(u-u_{k \Delta}\right)\right\rangle & =p A_{e}\left(\langle\sin \rangle-\left\langle\sin _{k \Delta}\right\rangle\right)+A_{e}^{2}\left(\left\langle\sin ^{2}\right\rangle-\left\langle\sin \cdot \sin _{k \Delta}\right\rangle\right) \\
& \simeq A_{e}^{2} \sin ^{2}(\pi f k \Delta)
\end{aligned}
$$

where $k$ indexes $u(t-k \Delta)$. We used $k=1$.

Phase reconstruction - similar to frequency we determined phase with an $O_{I I}$ dipolar moment taking reference to a fixed phase sine:

$$
\langle u \cdot \cos \rangle \simeq \frac{1}{2} A_{e} \sin (\phi)
$$

where for $\phi=0$ the error may be significant, however phase is not absolute, rather relative to the previous sample's phase, as such such errors tend to systematically cancel out. 


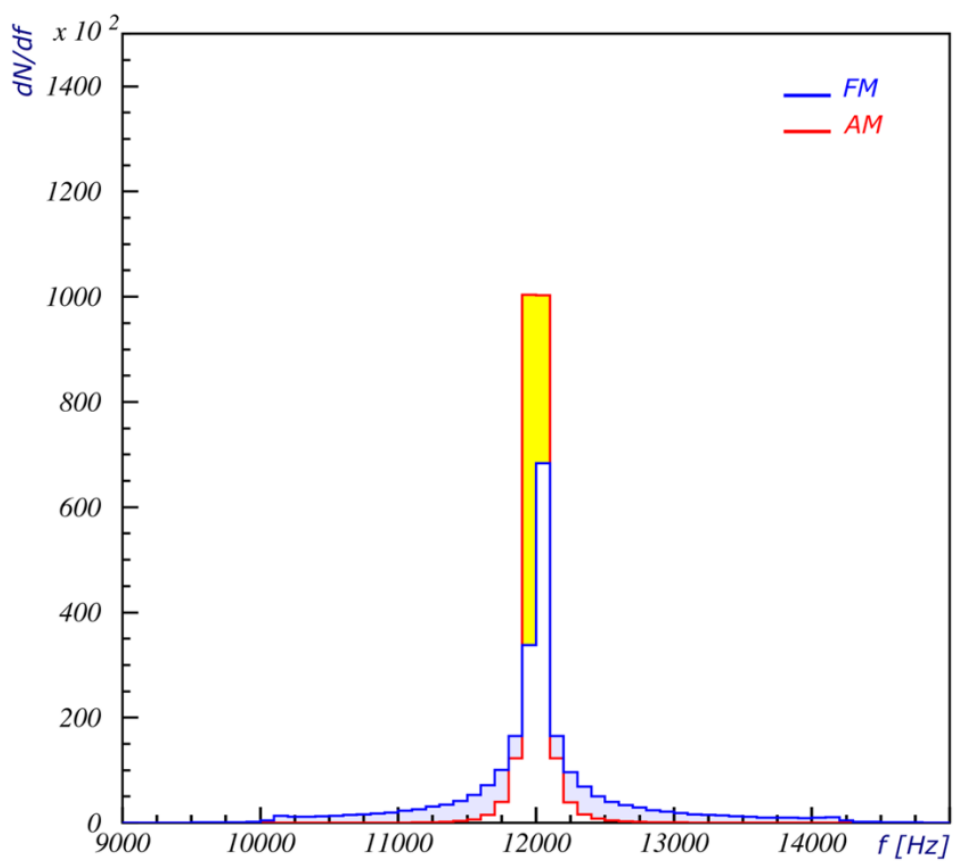

Figure 1. Distribution $d N / d f$ of number of samples with a given (intermediate) frequency. AM modulation (red) is evidently narrower in band, vs. FM modulation (blue). Thus the FWHM and the tail length are features of this histogram.

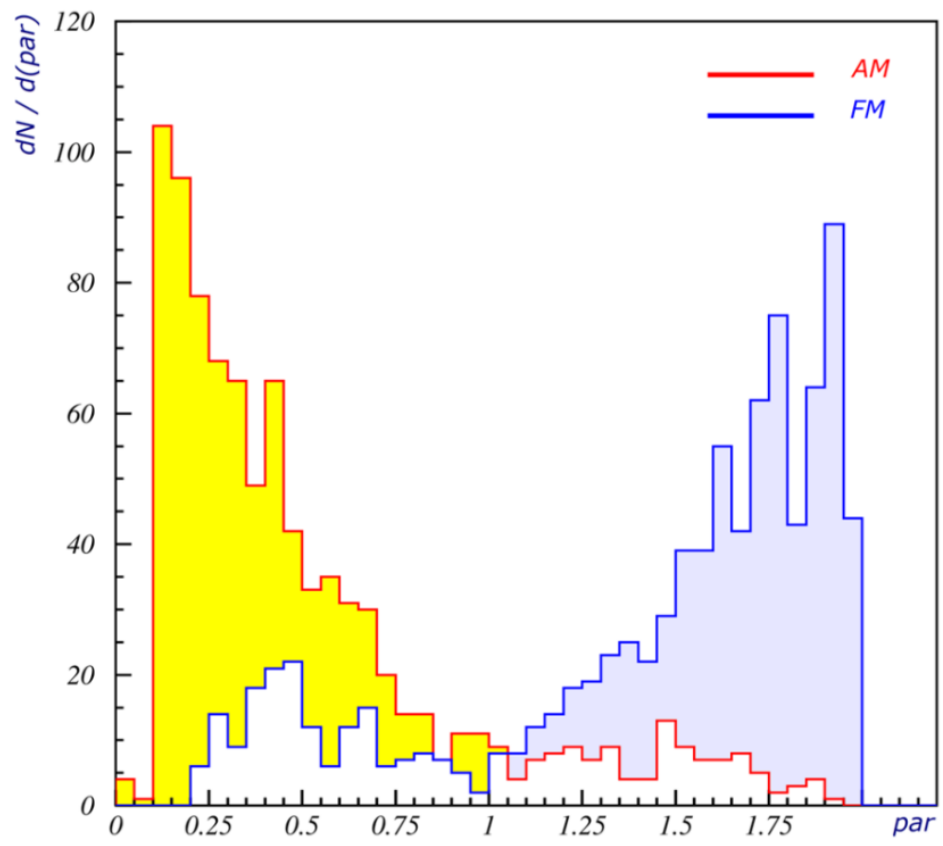

Figure 2. Distribution of number of histograms for the full width at $1 / 4$ maximum for the phase distribution histograms - with red AM modulation and blue FM modulation. Yellow represents AM excess over FM and light blue excess of FM over AM.

Feature creation - having pedestal, amplitude, frequency and phase for each sample, we accumulated these quantities in histograms looking for discriminating features. We devised similar other parameters (in number of 12) to capture the differences between various modulation types. As an 
example, the figure above shows the distribution of the full width at $1 / 4$ maximum for the phase distribution histograms - with red, again, AM modulation and with blue, FM modulation.

\section{Deep-learning performance and conclusions}

Using this set of 12 parameters we trained (with BFGS - Broyden-Fletcher-Goldfarb-Shanno algorithm for AM vs. FM, or stochastic for AM-LSB vs. AM-USB) deep learning neural networks for binary sets of modulations.

The results were excellent for instance AM vs. FM, and the most difficult case (and notable result too) was AM-LSB vs. AM-USB which are essentially the same type of modulation, and is known to be practically impossible to discern with the classical statistical-moments method. The figure below shows this result.

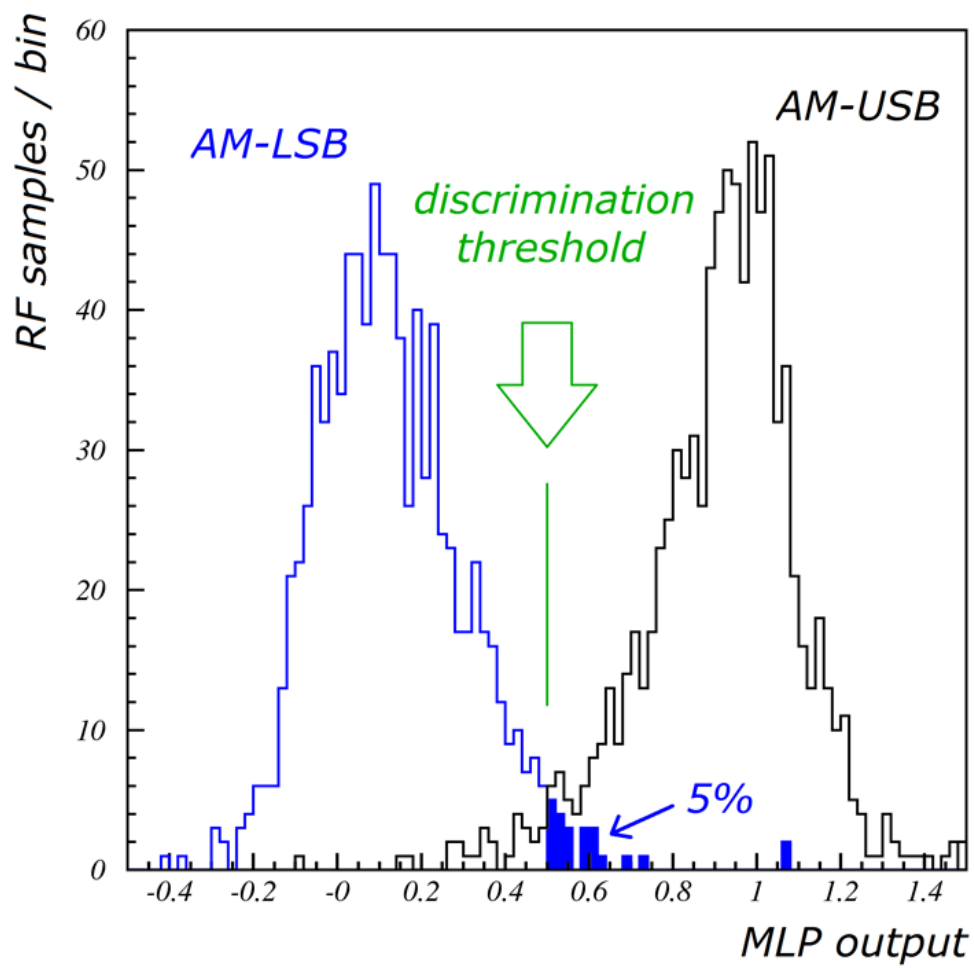

Figure 3. Showcase result, featuring the difficult case of discriminating AM-LSB vs. AM-USB modulation. The neural net output above 0.5 signals AM-USB (95\% correct flags) and below AMLSB. Given the similarity of the two modulations this is a remarkable result. The training method was stochastic-training.

Concluding, our deep learning neuromorphic software approach to AMC's confirms the promising results of a pleiad of other such approaches. What distinguishes our approach is a 2 step feature creation method. The first step reconstructs the basic signal parameters, pedestal, amplitude, frequency and phase and creates histograms with 1000 such samples (each $n=11$, "magic number" samples long). The second step analyses these histograms and draws parameters out of them. We found these parameters to be much better performing than the primary raw parameters.

As showcase for this conclusion stands the difficult case of discriminating AM-LSB vs. AMUSB modulation, which flags the correct modulation in $95 \%$ of cases. 


\section{References}

[1] J.L. Xu, W. Su, M. Zhou, Likelihood-Ratio Approaches to Automatic Modulation Classification, IEEE Trans. Syst. Man Cybernet. Part C Appl. Rev. 41, 455-469 (2011).

[2] A. Hazza, M. Shoaib, S. A. Alshebeili, A. Fahad, An overview of feature-based methods for digital modulation classification, Communications, Signal Processing, and their Applications, ICCSPA 1st International Conference IEEE, document 6487244, pp. 1-6, (2013).

[3] EE. Azzouz, AK. Nandi, Automatic identification of digital modulation types, Signal Process. 47, 55-69 (1995); JJ. Popoola, R. van Olst, A novel modulation sensing method, IEEE Veh. Tech. Mag. 6, pp. 60-69, (2011).

[4] J. Liu, Q. Luo, A novel modulation classification algorithm based on daubechies5 wavelet and fractional fourier transform in cognitive radio, 14th IEEE ICCT Communication Technology Conference, document/6511199, pp. 115-120, (2012); Y. Lv, Y. Liu, F. Liu, J. Gao, K. Liu, G. Xie, Automatic modulation recognition of digital signals using CWT based on optimal scales, IEEE Conference On Computer and Information Technology, document/6984692, pp. 430-434, (2014).

[5] D. Das, A. Anand, P. K. Bora, R. Bhattacharjee, Cumulant based automatic modulation classification of QPSK, OQPSK, $\pi / 4-Q P S K$ and 8-PSK in MIMO environment, IEEE Conference On Signal Processing and Communications, document/7439996, pp. 1-5, (2016); A. Hazza, M. Shoaib, A. Saleh, A. Fahd, Robustness of digitally modulated signal features against variation in HF noise model, EURASIP J. Wirel. Commun. Netw. 2011, 24, (2011).

[6] U. Satija, M. Manikandan, B. Ramkumar, Performance study of cyclostationary based digital modulation classification schemes, IEEE Conference on Industrial and Information Systems, document/7036609, pp. 1-5, (2014).

[7] M. W. Aslam, Z. Zhu, A. K. Nandi, Automatic modulation classification using combination of genetic programming and KNN, IEEE Trans. Wirel. Commun. 11, 2742-2750, (2012).

[8] K. Hassan, I. Dayoub, W. Hamouda, M. Berbineau, Automatic modulation recognition using wavelet transform and neural network, IEEE Conference on Intelligent Transport Systems Telecommunications, document/5399351, pp. 234-238, (2009).

[9] V. Orlic, M. Dukic, Multipath channel estimation algorithm for automatic modulation classification using sixth-order cumulants, Electron. Lett. 46, 1348-1349, (2010).

[10] GJ. Mendis, J. Wei, A. Madanayake, Deep learning-based automated modulation classification for cognitive radio, IEEE Conference on Communication Systems, document/7833571, pp. 1-6, (2016).

[11] A. Dai, H. Zhang, H. Sun, Automatic modulation classification using stacked sparse autoencoders, IEEE Conference on Signal Processing, document/7877834, pp. 248-252, (2016). 